\title{
Le champ d'action réticulaire : un espace de communication stratégique hybride
}

\section{Christian Marcon}

\section{(2) OpenEdition}

1 Journals

Édition électronique

URL : http://journals.openedition.org/communicationorganisation/2581

DOI : 10.4000/communicationorganisation.2581

ISSN : $1775-3546$

Éditeur

Presses universitaires de Bordeaux

Édition imprimée

Date de publication : 1 novembre 2001

ISSN : 1168-5549

Référence électronique

Christian Marcon, « Le champ d'action réticulaire : un espace de communication stratégique hybride », Communication et organisation [En ligne], 20 | 2001, mis en ligne le 27 mars 2012, consulté le 19 avril 2019. URL : http://journals.openedition.org/communicationorganisation/2581 ; DOI : 10.4000/ communicationorganisation.2581

Ce document a été généré automatiquement le 19 avril 2019

(c) Presses universitaires de Bordeaux 


\title{
Le champ d'action réticulaire : un espace de communication stratégique hybride
}

\author{
Christian Marcon
}

1 Le propos de cet article est de poser un jalon dans l'analyse de l'interaction entre les stratégies réseaux dont nous faisons notre champ de recherche central et la dynamique de communication à l'œuvre dans le cadre de telles stratégies. Nous qualifions de stratégie réseau toute stratégie qui consiste à créer ou, le plus souvent, à activer et orienter les liens tissés entre des acteurs au service d'un projet ${ }^{1}$.

D'un point de vue systémique, les champs complexes dans lesquels sont conduites des stratégies réseaux sont ouverts. Pour autant, cela ne signifie pas que tout réseau ait une capacité d'action infinie. De multiples variables contribuent à borner les théâtres d'opérations stratégiques, au premier rang desquelles nous situons la représentation, par les membres de tout réseau, des limites de leur potentiel d'action collective. Ainsi les champs d'action réticulaires sont-ils des construits d'action collective imaginaires, que le hasard des circonstances contribue à façonner au même titre que l'analyse stratégique des acteurs, les évolutions géostratégiques survenues après l'attentat perpétré à New York contre les tours du World Trade Center en sont un remarquable exemple. Au sein de leurs imaginaires collectifs, les acteurs ${ }^{2}$ développent des stratégies réseaux en jouant sur leurs maillages relationnels et institutionnels. Leur implication active participe à son tour, suivant un processus récursif et dialogique, au processus d'invention dynamique permanente de la réalité ${ }^{3}$ du champ d'action réticulaire.

3 Le rôle de la communication dans ce processus est à l'évidence essentiel tant la communication est intime au réseau. Toute stratégie réseau implique en effet une interaction entre les acteurs difficilement envisageable hors de la communication. Mais l'évidence s'arrête là dès que le problème est posé en termes stratégiques. De même que la simple appartenance à un champ d'action collective ne suffit pas pour affirmer l'existence d'un réel maillage relationnel entre des acteurs ${ }^{4}$, le constat de l'existence d'un 
tel maillage ne permet pas de présumer de l'existence d'une stratégie réseau. L'étude des flux de communication qui traversent le champ d'action collective ne saurait donc rendre suffisamment compte des logiques de communication mises en œuvre dans le cadre d'une stratégie réseau d'où émerge un contexte particulier d'expression du discours.

La première partie de cet article nous conduira à préciser la notion et les caractères du champ d'action réticulaire - héritier hybride du construit d'action collective de Crozier et Friedberg - qui constitue le cadre de référence de notre étude des dynamiques de stratégie réseau. Nous montrerons dans une deuxième partie comment la logique de constitution du réseau d'acteurs influe de manière semble-t-il déterminante sur la communication entre les acteurs, à destination de leur champ d'action réticulaire voire au-delà. Enfin, nous nous attacherons dans une dernière partie à analyser les principaux rôles dévolus aux technologies de l'information et de la communication dans les projets reposant sur une stratégie réseau.

\section{Du construit d'action collective au champ d'action réticulaire : une logique d'hybridation}

\section{La notion de champ d'action réticulaire}

5 En 1977, M. Crozier et E. Friedberg consacraient un ouvrage à l'étude sociologique des construits d'action collective ${ }^{5}$ constitués par des acteurs qui poursuivent, chacun, des objectifs divergents, voire contradictoires mais néanmoins parviennent à organiser des modes d'intégration qui assurent [leur] nécessaire coopération sans supprimer leurs libertés. Les auteurs mettaient en exergue l'immixtion du pouvoir dans toute action sociale, le caractère contingent de la structuration des interactions au sein de chaque construit, un double processus de création collective et d'apprentissage collectif à l'œuvre tant dans la résolution des conflits que dans l'évolution de cet espace social.

6 Crozier et Friedberg s'intéressaient ainsi à un espace d'interactions, forme de système collectivement élaboré, dans lequel l'individu n'est ni parfaitement et rationnellement autonome, ni strictement assujetti à la force du groupe. Un tel système ${ }^{6}$ nait, évolue et, parfois, disparait.

7 Le construit d'action collective de Crozier et Friedberg est un champ social dans lequel l'interaction entre les individus s'appuie sur des modes de communication que nous nous autoriserons à qualifier simplement de "traditionnels »: communication en face à face par la parole, les gestes, les mimiques, communication intermédiée via le courrier postal, le téléphone, le télex et la télécopie pour ce qui est des échanges individualisés, via la radio et la télévision pour ce qui concerne la communication de masse.

8 Crozier et Friedberg, concentrés sur d'autres problématiques, n'ont pas particulièrement analysé l'impact de ces modes de communication sur les construits d'action collective. D'autres chercheurs ont montré comment le simple bouche à oreille permit, et permet encore, la diffusion rapide d'informations à des groupes d'individus plus ou moins nombreux, propageant ainsi des imaginaires collectifs. Et puis chaque innovation technologique en matière d'outils de communication a offert de nouvelles possibilités de création et de diffusion d'imaginaires collectifs. L'Histoire se souvient, par exemple, du début de panique causé aux États-Unis par la pièce radiophonique d'Orson Wells décrivant l'arrivée des martiens sur la Terre. 
9 L'étude des stratégies réseaux révèle comment ceux qui les conduisent ont su tirer profit des nouveaux outils de communication mis à leur disposition. Le téléphone, en particulier, a favorisé à la fois l'extension territoriale du champ d'action des réseaux et leur rapidité de mobilisation.

10 Dans le contexte des outils de communication actuels, et sans tomber dans le travers « du tout technologique", une étude des construits d'action collective et des stratégies réseaux qui s'y opèrent perdrait toute pertinence en ignorant le «cybermonde ». Si l'intelligence et les objectifs restent avant tout ceux de l'homme, les capacités informatiques et la puissance des supports de communication génèrent de nouveaux potentiels stratégiques. Ce qui est radicalement nouveau, c'est l'enchevêtrement de l'humain et du technique. Dans le domaine de l'entreprise, W. Turner en décrit les conséquences stratégiques : « Les investissements dans [les technologies de l'information et la communication] sont en train de créer une situation organisationnelle que nous proposons de caractériser sous le nom de réseaux hybrides d'intelligence (les RHI). En effet, il nous semble d'ores et déjà possible de considérer l'entreprise comme un ensemble d'hommes et de machines travaillant en coopération sur un réseau informatique. Les RHI permettent d'envisager la gestion décentralisée des activités opérationnelles avec une centralisation rapide des informations nécessaires aux décisions stratégiques. $»^{7}$

11 Au regard de la diffusion rapide des technologies de l'information et la communication [TIC] dans la population au-delà du seul contexte du travail en entreprise, il apparaitt désormais que l'ensemble des construits collectifs est en phase de transition vers ce type de réseau hybride, à des rythmes certes extrêmement variables selon les tranches de population. En janvier 1999, Jo Link-Pezet écrivait ainsi : «La communauté scientifique intègre, dans un même espace, réseaux humains et réseaux de communication, avec leurs capacités respectives à produire de l'information, à communiquer et à coordonner en temps réel de l'intelligence distribuée dans un flux constant d'informations nouvelles. ${ }^{8}$ "

12 Ce mouvement d'ensemble ne rend pas obsolètes les travaux de Crozier et Friedberg. Tous les construits imaginaires ne procèdent pas de l'usage d'Internet. Dans l'affaire récente des attentats contre les tours du World Trade Center, ce sont les chaînes de radio et de télévision qui ont sans doute eu le plus fort impact de diffusion d'un imaginaire partagé. De même, les stratégies réseaux aujourd'hui les plus efficaces n'ont pas forcément recours à l'Internet. Toutefois, de nouvelles perspectives se font jour. Le terrain dans lequel opère et communique le stratège réseau se caractérise de plus en plus par une géographie hybride complexe, combinaison virtuelle d'acteurs et de supports de communication reliant ces acteurs. L'ensemble constitue ce que nous appelons un champ d'action réticulaire, afin, tout à la fois, de ne pas accentuer la polysémie du terme réseau et de signifier la dimension stratégique de notre approche.

\section{Les caractères fondamentaux du champ d'action réticulaire.}

13 Les circonstances qui président à la création de champs d'action humains sont extrêmement variables. Il serait vain d'essayer d'en dresser un inventaire. Néanmoins, passée la phase initiale parfois chaotique, les champs d'action humains tendent à se structurer. Ils s'inventent des modes d'intégration complexes [lois, traditions, normes de comportement, valeurs...] qui rendent possible l'existence durable du collectif. Le tout jeune cyberespace lui-même est structuré par un ensemble de réglementations, de 
déontologies d'entreprises et par la netiquette, ce code du savoir-vivre dont les internautes on jugé nécessaire de se doter ${ }^{9} . .$.

Ce principe général a pour implication stratégique que la création d'un réseau d'acteurs procède d'une logique de greffage. Le stratège réseau s'efforce d'implémenter la configuration souple de liens capable de faire aboutir son projet dans un champ d'action réticulaire choisi, en se référant à la structure préexistante de celui-ci. On ne crée pas un réseau sans tenir compte des liens et des rapports de pouvoir préexistants. Dans le domaine des entreprises, par exemple, fédérations et clubs s'organisent, chacun à leur manière : la fédération accueille toute entreprise qui appartient au champ professionnel qu'elle couvre ; a contrario, le club d'entreprises se constitue selon une logique sélective : les membres se choisissent en fonction de leurs positions dans le champ d'action. Il en va de même de la constitution de réseaux d'affinité ${ }^{10}$ entre individus. Les strates sociales qui structurent une ville, par exemple, favorisent les réseaux infra strate et découragent la constitution de réseaux inter strates. Un phénomène auto entretenu par les membres de ces strates : les parents appartenant à une catégorie socioprofessionnelle aisée déploient des trésors de stratégie réseau, jouant de leur réseau de "relations» pour obtenir l'inscription de leurs enfants dans les établissements scolaires fréquentés par les enfants de familles aisées. De ce point de vue, le champ d'action réticulaire neutre n'existe pas.

Nous admettons avec Crozier et Friedberg que les construits humains sont « irréductiblement contingents, c'est à dire non déterminés » et que " ni leur création ni leur évolution ne reposent sur aucune loi universelle, sur aucune nécessité ou tendance historique $^{11}$ ». Il est illusoire de rechercher un quelconque modèle universel de réseau, mieux prédisposé que d'autres à la conduite d'une stratégie. La culture et les représentations propres à chaque champ d'action réticulaire induisent des formes possibles de réseau, supports de la stratégie. Certains modèles sont connus: famille, association, club, fédération, alliance, groupement informel... Mais les réseaux humains ne se décrètent pas. ${ }^{12}$ Réseau en étoile, réseau en $Y$, réseau non centralisé ${ }^{13}{ }^{3}$ 'avèrent, tour à tour, plus ou mois efficaces pour atteindre l'objectif stratégique. Il revient au stratège d'identifier la forme du réseau la mieux adaptée à son champ d'action et d'analyser la façon dont, par la communication et l'information, il peut exercer une action de nature à faire évoluer le réseau dans ses décisions, dans ses comportements, dans son imaginaire partagé... Sur ce point, l'observation d'un grand nombre de réseaux professionnels et personnels révèle que le stratège est fortement tributaire de la logique territoriale ou thématique qui préside à la constitution du réseau.

\section{Logique de constitution du réseau et discours des stratèges}

\section{Les discours de l'appartenance territoriale}

La première logique de constitution des réseaux d'acteurs est leur inscription dans un espace géographique, leur territoire d'appartenance, réel autant de symbolique. Les médias se font ainsi régulièrement l'écho du phénomène des «bandes » constituées dans des quartiers par des jeunes qui se créent, dans cet espace qu'ils s'approprient, un mode de communication commun reposant sur un vocabulaire spécifique, sur des codes vestimentaires, sur des comportements partagés... Lorsque les relations à la fois souples et structurées qu'ils créent sont mises au service d'un projet, alors se mettent en branle 
de véritables stratégies réseaux. Dans le domaine économique, les analyses ont montré le bénéfice que les entreprises retirent de leur appartenance à un territoire. Lorsque l'intimité de leur activité avec le territoire devient étroite au point que leurs destins semblent inextricablement liés, la communication au sein de ce territoire en est fortement affectée.

17 L'observation montre que, conformément à l'affirmation de Von Foerster selon lequel «l'environnement, tel que nous le percevons, est notre invention ${ }^{14}$, les réseaux, et au premier rang d'entre eux les réseaux territorialisés, développent naturellement un imaginaire collectif ${ }^{15}$ partagé : histoire des grandes heures du territoire et de ses héros, entreprises mythiques, sentiment d'un destin commun, perception des territoires environnants... Cet imaginaire collectif, espace mouvant qui n'est pas pure subjectivité, imprègne le dit et le non-dit, diffusant à travers toute la communication, à usage interne ou externe. Dans une dynamique d'auto-renforcement, les réseaux en viennent parfois à s'imiter en se projetant eux-mêmes comme modèles au travers de leur communication ${ }^{16}$. Les grands stratèges réseaux utilisent parfaitement dans leurs discours le potentiel qu'offre cet imaginaire qui les affecte eux-mêmes, comme une force intégrative entre les membres $^{17}$. Il tient parfois lieu de raison d'être pure et simple du réseau, comme le montrent certains réseaux terroristes nationalistes. Plus généralement, la création d'un hiatus entre l'imaginaire développé par le réseau, auto alimenté par son discours, et la réalité de l'existant, constitue le premier pas vers un affaiblissement stratégique par manque de lucidité.

\section{Le cas complexe des champs d'action thématiques}

L'existence d'une affinité thématique, d'une proximité d'intérêt entre des acteurs constitue l'autre soubassement de la constitution de réseaux. Supporters d'une équipe, fans d'un artiste, mais aussi membres d'une même profession, défenseurs des mêmes idées politiques, religieuses, écologiques, sociales ou encore chercheurs dans un même domaine se rencontrent à l'occasion de manifestations dédiées au thème transterritorial qui crée leur affinité et, désormais, participent aux mêmes forums de discussion. Cette proximité thématique génère une connivence. De la connivence naissent les liens. Ces liens, lorsqu'ils perdurent, fondent des réseaux, ouvrant ainsi des espaces stratégiques.

Dans la vie sociale, l'affinité thématique s'accompagne couramment d'une proximité émotionnelle. Il n'est que de voir les passions déchaînées par un match de football dans l'enceinte d'un stade peuplé d'amoureux de ce sport. Dans un tout autre domaine, l'imaginaire raciste repose un peu sur une analyse de ce qui est supposé différencier et opposer les races et beaucoup sur des mouvements pulsionnels et passionnels.

Dans le monde des entreprises, la proximité thématique se double fréquemment, mais pas nécessairement, d'une interdépendance économique, verticale dans le cadre de filières, ou horizontale, à un même stade du processus de production et ce, quels que soit le territoire d'implantation, mondialisation oblige. La communication développée par ces réseaux est directement affectée par cette proximité. Dans la logique horizontale, l'échange d'informations entre les membres souffre souvent de la concurrence qui ne manque pas de s'exercer entre eux. En revanche, la logique verticale, dans laquelle les acteurs sont davantage complémentaires que concurrents, favorise l'échange d'informations. 
21 Naturellement, la réalité stratégique est beaucoup plus complexe que ces analyses séparées des champs territoriaux et thématiques ne pourraient le laisser entendre. Bien souvent, les stratèges réseaux agissent à l'intersection des deux types de champs, ce qui rend plus complexe leur pilotage stratégique, et plus difficile le décryptage des logiques de communication à l'œuvre.

\section{Les technologies de l'information/communication au cœur de la stratégie réseau}

Il serait prématuré de tirer des conclusions définitives sur l'impact de l'intimité encore récente des réseaux d'acteurs et des TIC. Comme l'écrit Serge Proulx, « en ce qui concerne les phénomènes techniques, [dans une perspective constructiviste] il y aurait un enchevêtrement dynamique, instable et permanent entre le pôle des technologies et celui de l'environnement social. En d'autres mots, tout objet ou dispositif technique porterait l'empreinte des rapports sociaux qui le sous-tendent et, en même temps, la dimension technique traverserait de plus en plus largement l'ensemble des formes sociales ${ }^{18}$. Cependant, l'influence de la dimension technique sur les stratégies réseaux est déjà perceptible à plusieurs niveaux.

23 L'irruption des TIC affecte le processus de communication et la relation même entre les membres du réseau à plusieurs niveaux.

\section{La revanche des petits}

L'étroite intégration des réseaux numériques et des réseaux d'acteurs conduit à la création de dispositifs dotés d'un potentiel stratégique redoutable. Mettre « un ensemble collaboratif de ressources informatiques et de transmission offrant des services de communication $^{19}$ » au service d'un réseau d'acteurs tendus vers la réalisation d'un projet partagé, c'est assurément lui donner des moyens d'action dont ne disposaient pas les réseaux quelques années en arrière.

La démocratisation de l'accès à ces moyens impose de repenser les questions stratégiques de la taille et de la localisation de l'acteur. Un internaute seul n'a-t-il pas réussi à inquiéter le géant de l'automobile Ford au moyen d'un site Internet relatant les problèmes de qualité rencontrés par de nombreux internautes? Le site « jeboycottedanone.com » n'a-t-il pas été considéré comme suffisamment dangereux pour que l'entreprise mise en cause juge nécessaire d'intenter une action en référé pour essayer de l'interdire ${ }^{20}$ ? Désormais, les réseaux numériques permettent à des tout petits groupes d'individus dispersés de se rassembler virtuellement autour d'une cause commune et de disposer, grâce à Internet, d'une audience importante ${ }^{21}$. Le potentiel de concentration virtuelle des forces offert par la «toile" pallie la dispersion spatiale des acteurs.

Capacité nouvelle à rassembler des forces très rapidement avec des efforts limités, bouleversement du cadre spatial, liberté d'action sur Internet: plus que jamais, l'outil met la communication au cœur des stratégies réseaux dont il redimensionne le champ d'action. 


\section{Une prise en compte à géométrie variable.} Elles sont alors au cœur de la constitution d'un réseau d'acteurs. C'est le cas de Grand Place, à Lille, par exemple qui se présente comme « un projet de messagerie électronique [qui] vise prioritairement à favoriser le développement de projets, faciliter l'échange d'informations et promouvoir de nouvelles formes de communication et d'échanges. Ce projet s'inscrit dans la démarche de la société de l'information et souhaite encourager l'émergence d'une communauté régionale des mondes économique, de l'enseignement, de la recherche et des institutions ${ }^{26}$ » La même volonté de placer le réseau de TIC au cœur du projet se retrouve dans « huit projets démontrant l'utilisation de nouvelles technologies dans le commerce électronique ${ }^{27}$ ", soutenus par la Direction Générale des Entreprises [DGE] de la Commission Européenne

31 Dans bien des cas, les TIC ne constituent pas le projet en lui-même mais sont utilisées par le stratège comme un moyen moderne de mobilisation. La multiplication des portails thématiques sur Internet donne une idée de la dynamique que peut susciter la conjugaison d'un thème concernant une communauté d'individus et de TIC porteuses d'une image gratifiante. Les TIC sont alors un détour stratégique au service d'un projet de collaboration en réseau plus ambitieux. Citons en exemple le projet TISS de développement d'un réseau d'entreprises textiles dans le $\mathrm{Gard}^{28}$, le projet multimédia du Programme Régional Ruralter ${ }^{29}$ visant à favoriser le développement rural en Amérique Latine, et celui de Cyberlomont ${ }^{30}$ à destination de la population du Pays de Lomont.

De plus en plus, la diffusion de l'usage des TIC crée une info structure, autrement dit une culture de communication commune aux membres du réseau. Ses caractéristiques 
générales sont désormais bien connues: simplicité de la forme du message, réponse rapide à tout message, règles de bonne conduite... Souvent génératrice d'une forme d'émulation, cette info structure modifie sensiblement le modus operandi de la communication dans le cadre de stratégies réseaux : réactivité accrue, interaction plus forte, partage des informations ${ }^{31}$ suscitent une maïeutique nouvelle dans la formulation du projet, sa reconfiguration, son pilotage... Le risque associé à cet usage des TIC est celui du renversement de la perspective stratégique : l'outil TIC cesse d'être un moyen pour devenir une fin en soi, absorbant toute l'énergie des acteurs au détriment du projet initial.

Lors de la renégociation de la convention de Lomé, la Commission Européenne a fait ainsi l'expérience de la mise en place d'un forum électronique afin d'accroitre le nombre d'acteurs participant à la réflexion et d'enrichir les échanges. A. Larregle, inscrite dans ce projet a décrit des conséquences de cette expérience sur l'infostructure de l'Equipe d'Animation du Débat Public : plus grande réceptivité à l'interactivité, sensibilisation à l'éthique, à la relation aux autres acteurs, prise de conscience de la nécessité des TIC, expérimentation de nouveaux formats de communication... ${ }^{32}$

Les TIC interviennent enfin à deux niveaux dans le processus d'apprentissage qui s'opère au sein des réseaux. Elles contribuent d'abord à son accélération en réduisant les délais de transmission des informations. Du point de vue stratégique, cette accélération du processus entraîne l'accélération parallèle de la boucle OODA [Observation - Orientation Décision - Action] ${ }^{33}$. Par là même, les réseaux recouvrent parfois une liberté d'action stratégique perdue ${ }^{34}$ Rappelons enfin, mais ce phénomène a déjà été fréquemment exposé par ailleurs, que les TIC élargissent aussi le processus d'apprentissage à de nouvelles compétences et de nouvelles connaissances.

\section{Conclusion}

35 L'approche de la problématique communication/stratégie réseau dans le contexte paradigmatique du champ d'action réticulaire nous a permis une première présentation de ce dernier, compris comme une combinaison virtuelle d'acteurs et de supports de communication reliant ces acteurs. Le champ d'action réticulaire est un construit d'action collective [Crozier \& Friedberg], dans lequel la relation homme-ordinateur se fait de plus en plus prégnante, aboutissant par là à la création dans tous les domaines de réseaux hybrides d'intelligence dont Turner a mis en évidence l'émergence dans le cadre organisationnel de l'entreprise. Au stade actuel de notre recherche, deux dimensions spécifiques de l'interaction communication/stratégie réseau apparaissent clairement : la relation entre la logique initiale d'ancrage du réseau (thématique versus territoriale) et les modes de communication mis en pratiques d'une part; la contribution polymorphe des TIC aux stratégies réseaux d'autre part.

$\mathrm{Ce}$ dernier point appelle, en conclusion, un questionnement. $\mathrm{Au}$ vu des multiples contributions possibles des TIC à la conduite de stratégies réseaux, devons-nous considérer que celles-là sont aujourd'hui tellement intimes à la conduite de celles-ci qu'elles leur sont dès maintenant indispensables? Pour être dans l'air du temps, sans doute faudrait-il répondre par l'affirmative. En convoquant les réseaux hybrides pour analyser nos champs d'action réticulaires, nous semblons aller dans ce sens. Néanmoins, force est de se garder de l'illusion du caractère indispensable des TIC dans toute stratégie réseau actuelle. 
Nous en voulons pour preuve le projet Epsilon, soutenu par la DGE de la Communauté Européenne, qui suit une quinzaine de clubs d'entreprises fondés sur les TIC. La DGE reconnaît qu'il existe un large éventail de réseaux d'entreprises fondés sur les TIC mais que trois obstacles doivent encore être surmontés avant de faire des TIC un réel support de stratégie : l'insuffisante maitrise des TIC par les chefs d'entreprises industrielles; le manque de méthodologie et d'outils pour manier la gestion de connaissances ; la lenteur de la constitution de réseaux solides au niveau de grappes d'entreprises. Or l'observation montre que les chefs d'entreprises, même néophytes en matière de nouvelles technologies, savent conduire des stratégies réseaux... En somme, il y a loin, encore aujourd'hui, de la coupe aux lèvres.

\section{BIBLIOGRAPHIE}

BATESON, G., RUESCH, J., Communication et société. Paris : Seuil, 1988. 347 p.

CASTELLS, M., Le pouvoir de l'identité. L'ère de l'information. Paris : Fayard, 1997, 538 p.

CROZIER, M., FRIEDBERG, E., L'acteur et le système. Les contraintes de l'action collective. Paris : Seuil, 1977, $493 \mathrm{p}$.

DEGENNE, A., FORSE, M., Les réseaux sociaux. Paris : Armand Colin, 1994, 288 p.

DEGENNE, A., FORSE, M., Comment on trouve ses amis. Enquête sur la sociabilité des français. Sciences Humaines, hors série n 5, Mai/juin 1994 FACHINELLI, A.C., MARCON, C, MOINET, N., Agilité ou paralysie stratégique : considérations réticulaires sur la boucle OODA. In $5^{\mathrm{e}}$ colloque FrancoBrésilien, 7-9 janvier 2001, Poitiers. 8 p.

FADOK, D. S., La paralysie stratégique par la puissance aérienne. Paris : Economica - Institut de stratégie comparée, Collection Hautes études stratégiques, $n^{\circ}$ 10, 1998.

FAYARD, P., MOINET, N., Quand le réseau est stratégie. Echanges n ${ }^{\circ} 108$, octobre 1994, p. 45 à 48

FAYARD, P., La maîtrise de l'interaction. Paris : 00h00, 2000

FLAMENT, C. Réseaux de communication et structures de groupes. Paris :

Dunod, 1965

FOERSTER (von), H., La construction d'une réalité. In WATZLAWITZ P., L'invention de la réalité. Contributions au constructivisme. Paris : Seuil, 1988,356 p.

GHERNAOUTI-HELIE, S., DUFOUR, A., De l'ordinateur à la société de l'information. Paris : Presses Universitaires de France, coll. Que sais-je ? 1999, 126 p.

HUSSERL, Recherches pour la phénoménologie et la théorie de la connaissance. Paris : Presses Universitaires de France, 1961 ISRAEL, R. et AUFFRET, G., Une mémoire de l'émergence : vers un outillage conceptuel et socio-technique de la coopération. Solaris, $n^{\circ}$ 5, 1998 JACQUES-GUSTAVE, P., MOINET, N., Intelligence économique et stratégie des PME. Etude Intelco-Labcis, 1995

LAMIZET, B., SILEM, A., Dictionnaire encyclopédique des sciences de l'information et de la communication. Paris : Ellipses, 1997, 590 p. 
LINK-PEZET, J., De la représentation à la coopération : évolution des approches théoriques du traitement de l'information. Solaris nº 5, janvier 1999.

MARCON, C, MOINET, N., La stratégie-réseau. Essai de stratégie. Paris : 00h00, novembre 2000, 234 p.

MARCON, C, Intelligence économique : L'environnement pertinent comme variable stratégique. Justification théorique et approche instrumentale. Thèse de doctorat, Poitiers, 1998, 670 p.

MARCON, C, Rencontres de Nîmes. Le réseau sous toutes ses coutures. Veille, juin 2000, n 35 , p. 28-29

MUCCHIELLI, A., Approche systémique et communicationnelle des organisations. Paris : Armand Colin, $1998,160 \mathrm{p}$.

PERSUY, F., David contre Goliath : les petits font trembler les grands. Web Magazine, mars 2000, p. 94-95

PROULX, S., La construction sociale des objets informationnels : matériaux pour une ethnographie des usages. Université du Québec, Montréal REVELLI, C, Intelligence stratégique sur Internet. Paris : Dunod, 1998, 215 p.

SFEZ, L., Dictionnaire critique de la communication. Paris : Presses Universitaires de France, t. 1, 1993, $922 \mathrm{p}$.

TURNER, W., Les réseaux hybrides d'intelligence : penser l'entrelacement de l'humain et du technique. Solaris $\mathrm{n}^{\circ} 1,1994$.

WATZLAWICK, P., La réalité de la réalité. Confusion, désinformation, communication. Paris : Seuil, 1978, $257 \mathrm{p}$.

Sciences Humaines, Hors série nº 16, mars-avril 1997

\section{NOTES}

1. C. Marcon \& N. Moinet, La stratégie-réseau. Essai de stratégie. Editions ZéroHeure, Paris, 2000

2. B. Lamizet et A. Silem définissent l'acteur comme « une personne qui accomplit un acte réel, et qui se fait reconnaître pour cette raison par les autres personnes de l'espace social» in Dictionnaire encyclopédique des sciences de l'information et de la communication, Editions Ellipses, Paris, 1997, p. 7 Dans nos travaux sur les stratégies-réseaux, nous avons choisi de retenir une définition plus large de l'acteur : est acteur toute entité capable de prendre une décision (penser) et de la mettre en cuvre (agir). Ceci inclut les individus, les entreprises, les associations, les institutions, les réseaux...

3. P. Watzlawick, La réalité de la réalité. Confusion, désinformation, communication. Editions du Seuil, Paris, 1978

4. Du point de vue stratégique, un maillage relationnel n'est qu'une matière première tant qu'il n'est pas activé et finalisé. Sur les réseaux sociométriques, les systèmes de relations et les "constellations de travaux de Mintzberg», voir A. Mucchielli, Approche systémique et communicationnelle des organisations, éditions Armand Colin, 1998, p. 16 et suiv.

5. M. Crozier \& E. Friedberg, L'acteur et le système. Les contraintes de l'action collective. Editions du Seuil, Paris, 1977. Les citations en italiques de ce paragraphe sont extraites de cet ouvrage.

6. Nous prenons en référence la définition du système proposée in L. Sfez, Dictionnaire critique de la communication. Editions PUF, tome 1, Paris, 1993, p. 215

7. W. Turner, Les réseaux hybrides d'intelligence : penser l'entrelacement de l'humain et du technique, Solaris $\mathrm{n}^{\circ} 1,1994$. 
8. Jo Link-Pezet, De la représentation à la coopération : évolution des approches théoriques du traitement de l'information. Solaris $n^{\circ} 5$, janvier 1999.

9. Sur ce sujet, consulter le site suivant : http ://strasbourg.linuxfr.org/ raphael/textes/usenet/ rfc 1855.html

10. Pour une présentation de la sociométrie des réseaux issue de l'approche de J.L. Moreno, voir A. Degenne et M. Forsé, Les réseaux sociaux. Editions Armand Colin, Paris, 1994. Pour une analyse stratégique plus développée, C. Marcon et N. Moinet, op. cit., pp. 118-127

11. Michel Crozier et Erhard Friedberg, op. cit. p. 33

12. Pascal Jacques-Gustave et Nicolas Moinet, Intelligence économique et stratégie des PME, Etude Intelco-Labcis, mai 1995

13. Sur ces points, voir par exemple C. Flament, Réseaux de communication et structures de groupes, Paris : Editions Dunod, 1965

14. H. von Foerster, La construction d'une réalité, in Paul Watzlawitz, dir., L'invention de la réalité, contribution au constructivisme. Editions du Seuil, Paris, 1988

15. C. Marcon, Intelligence économique: L'environnement pertinent comme variable stratégique. Justification théorique et approche instrumentale, Thèse de doctorat, Université de Poitiers, 1998, ch. 2

16. Il nous semble pouvoir transposer ici en toute rigueur la formule de R. Israël et G. Auffret : «En somme, l'homme s'imite en se projetant lui-même comme modèle.» in Une mémoire de l'émergence : vers un outillage conceptuel et socio-technique de la coopération, Solaris, n5, 1998 17. «Il est évident que nous ne pouvons pas juger sans nous représenter l'état des choses sur lequel nous jugeons. » E. Husserl, Recherches pour la phénoménologie et la théorie de la connaissance. Edition P.U.F, 1961. $3^{\mathrm{e}}$ édition, 1993, p. 248

18. S. Proulx, La construction sociale des objets informationnels: matériaux pour une ethnographie des usages, Université du Québec à Montréal

19. S. Ghernaouti-Hélie, A. Dufour, De l'ordinateur à la société de l'information, Paris, Presses Universitaires de France, coll. Que sais-je ?, 1999, pp. 74-75

20. Voir par exemple : http ://www.net-iris.com/watch/press/ ?date=18-05-2001 \#1364

21. F. PERSUY, David contre Goliath: les petits font trembler les grands, Web Magazine, mars 2000, pp. 94-95

22. http ://www.oecd.org/sah/activities/PASP/index-fr.htm

23. Envie Développement est un réseau associatif d'entreprises d'insertions autonomes qui, sous la marque Envie, vendent des appareils électroménagers d'occasion.

24. J. Holc, Animation d'un réseau national de membres, compte-rendu d'expérience de l'introduction des TIC dans le fonctionnement du réseau Envie.

http ://www.fdf.org/fr/actions/fiche3 seminaire.html

25. P. Fayard \& N. Moinet, Quand le réseau est stratégie, Echanges n 108, octobre 1994, p. 45 à 48

26. http ://www, infonord.org/histGP. htm

27. Solon Consultants, Projets démontrant l'utilisation de nouvelles technologies dans le commerce électronique. Direction Générale des Entreprises, Commission Européenne, juillet 1999

28. C. Marcon, Rencontres de Nîmes. Le réseau sous toutes ses coutures, Veille $\mathrm{n}^{\circ}$ 35, juin 2000 , pp. 28-29

29. http ://www.fdf.org/fr/actions/fiche6 seminaire.html

30. http ://www.pays-du-lomont.com/proiet/cvberlomont/cyberlomont.html

31. C. Revelli, Intelligence stratégique sur Internet, Editions Dunod, Paris, 1998, p. 38

32. http://www.ue-acp.org et http ://www.ue-acp.org/fr/index.html

33. D. S. Fadok, La paralysie stratégique par la puissance aérienne, Paris, Economica Coll. Hautes études stratégiques, $\mathrm{n}^{\circ} 10,1998$.

34. A. C. Fachinelli, C. Marcon, N. Moinet, Agilité ou paralysie stratégique : considérations réticulaires sur la boucle OODA, Vième colloque Franco-Brésilien, janvier 2001, Université de Poitiers 


\section{RÉSUMÉS}

À partir de l'analyse des construits d'action collective réalisée par M. Crozier et E. Friedberg, cet article introduit d'abord le concept de champ d'action réticulaire, espace dans lequel se développent des stratégies-réseaux. Deux logiques aboutissent communément à l'émergence de champs d'action réticulaires : une logique territoriale et une logique thématique. L'influence de ces logiques sur les discours des stratèges-réseaux est analysée. Constatant enfin le processus en cours par lequel les réseaux évoluent vers des hybrides homme-machine, l'article s'interroge, à partir d'un ensemble de cas observés, sur l'impact des technologies de l'information et de la communication sur les stratégies-réseaux.

Considering the analysis of M. Crozier and E. Friedberg about the figures of collective action, this article proposes the new concept of area of network action, in which network-strategies usually spread. Area of network actions seems to have two origins in general. The first one is to belong to a specify territory. The second one is a common interest in a particular subject. Both origins influence mostly the internal and external communication of the networks. Last but not least, the new technologies of communication seem to have various impacts on networks. The ways networks are transforming in hybrid man and machine networks are analyzed.

\section{INDEX}

Mots-clés : champ d'action, construit d'action collective, imaginaire, réseau, stratégie

\section{AUTEUR}

\section{CHRISTIAN MARCON}

Christian Marcon est enseignant en communication, marketing, stratégies et réseaux à l'Institut de la Communication et des nouvelles Technologies [ICOMTEC] de l'université de Poitiers. Membre du LABCIS de cet institut [Laboratoire de Recherche en Communication et Information Scientifique et Technique], ses recherches portent sur les problématiques de stratégies-réseaux : épistémologie, impact des TIC, dynamiques stratégiques et de communication, dimensions organisationnelle et managériale. Il est l'auteur, avec Nicolas Moinet, de l'ouvrage La stratégie-réseau. Essai de stratégie. 